\title{
On Calculating the Eigenvalues of the Finite Hill's Differential Equation
}

\author{
Ekkehard Wagenführer
}

Universität Regensburg, Universitätsstraße 31, D-8400 Regensburg (Fed. Rep.)

\begin{abstract}
Summary. Some methods for evaluating the characteristic exponents in connection with Newton's iteration are applied for solving the eigenvalue problem related to the finite Hill's differential equation or, in particular, Mathieu's equation. By using these methods a high accuracy is achieved, furthermore a complete error analysis, which yields rather realistic error bounds, is possible.
\end{abstract}

Subject Classifications: AMS(MOS): 65L15 CR: 5.17.

\section{Introduction}

The characteristic exponent $v$ of the finite Hill's differential equation

$$
y^{\prime \prime}(x)+(\lambda+q(x)) y(x)=0
$$

with $\lambda \in \mathbb{C}$ and

$$
q(x)=\sum_{\kappa=1}^{k}\left(2 t_{\kappa}\right) \cos (2 \kappa x) \quad\left(t_{\kappa} \in \mathbb{C}\right)
$$

is defined by the existence of a solution $y \neq 0$ of (1) with

$$
y(x+\pi)=e^{i \pi v} y(x) \quad(x \in \mathbb{R}) .
$$

The characteristic exponent can be evaluated by the numerical solution of the differential equation, on the one hand. For this, according to [24], the Taylor method of high order has proved to be most convenient. On the other hand there are relations between $v$ and certain infinite determinants. The originally slow convergence of these determinants is accelerated by splitting up certain infinite products. For the general case of the finite Hill's equation, such a method has been given by Mennicken in [10] and discussed by the author in [23] under numerical aspects. In the special case of Mathieu's differential equation these methods coincide with those given by Schäfke and Schmidt 
[15]: a method which converges even more rapidly has been developed by the author in [22].

The present paper treats the numerical solution of the inverse problem, called the eigenvalue problem, which consists in finding $\lambda$ so that a given $v \in \mathbb{C}$ becomes a characteristic exponent of (1). The eigenvalues related to $v=0$ or 1 are of particular interest: for these there are $2 \pi$ - (or $\pi$-)periodic solutions of (1); furthermore, in the real case these $\lambda$-values mark the endpoints of the instability intervals. Whereas this problem for the general finite Hill's equation is rarely treated in the literature (cf. [13]), for Mathieu's equation several methods are known. Some of these are iteration methods founded on the wellknown continued-fraction relations quoted by Meixner and Schäfke [9], p. 117: A direct iteration method, the convergence of which is not proved in general, has been used by Tamir [18] for evaluating some eigenvalues related to $v=0.1,0.2, \ldots, 0.9$; other iteration methods, which are at least locally convergent, are given by Bouwkamp [3] - see also [9], p. 216 - and Arscott et al. [1]. In these papers no error analysis is presented.

For the Ritz-Galerkin method the Sturm-Liouville eigenvalue problem related to (1) is replaced by a finite-dimensional problem: from experience this method yields an accuracy sufficient for graphical purpose even in case of small dimensions (see Petersen [13]). For $v=0,1$ in the real case the authors of [19] have carried out an error analysis. According to Weinstein and Stenger [26] and Fichera [4] some other methods of constructing upper and lower bounds on the eigenvalues are based on inclusion theorems concerning positive compact operators in a Hilbert space. These methods have been developed in view of partial differential equations. They have been applied to some numerical examples of Mathieu's differential equation (in the real case, with $v=0,1$ ), for demonstration: these methods are rather expensive, their order of convergence is not known. According to a theorem of Veltkamp and van der Krogt [21], under certain assumptions any eigenvalue of an infinite tridiagonal matrix can be interpreted as a zero of the characteristic polynomial of a cut-off $(N, N)$-matrix, the $(N, N)$-coefficient of which has been replaced by a continued fraction depending on $\lambda$. For computing such a zero in the real case the bisection method or regula falsi can be used. By this means Jansen and Jeuken [6] and Thurlings [20] have programmed the calculation of eigenvalues of the real Mathieu's equation for $v=0,1$ : the Lamé equation can be treated in a similar way according to Jansen [5].

Here we deal with the eigenvalue problem for any given $v$ and in the complex case: for simplicity the rounding-error analysis is carried out only in the real case. The foundations are the methods for calculating the characteristic exponents according to [22-24], in connection with Newton's method. In the first section we list the functions the zeros of which have to be computed. Furthermore, we give an error estimate for Newton's method. The a priori estimations of the solutions of (1), which are given in Sect. 2, are needed in several parts of the error analysis: they are based on a generalization of Banach's fixed-point theorem given by Schäfke [14], applied to a quasi-metric mentioned by Schröder [16]. In Sect. 3 Newton's method is treated in con- 
nection with the numerical solution of the differential Eq. (1). Unlike the usual realization of shooting methods (cf. [17], p. 163) the derivatives with respect to $\lambda$ are evaluated by the numerical solution of the related inhomogeneous differential equation. The error analysis from [24] is slightly improved and generalized for the inhomogeneous problem. Finally the asymptotic behaviour of the error bounds related to increasing $\lambda>0$ and simultaneously decreasing step-width $h$ is discussed.

In Sect. 4 we restrict ourselves to Mathieu's differential equation: from the determinantal method presented in [22] we develop an algorithm of the same order for evaluating the derivatives with respect to $\lambda$. Again, a complete error analysis including the rounding errors is performed.

As the numerical examples in Sect. 5 show, the presented methods are favorable if high accuracy is required; in particular, the determinantal method for Mathieu's equation yields excellent error bounds over a wide range of the parameters.

The algorithms given in the Sects. 3 and 4 for evaluating the derivatives will yield more accurate values than needed for Newton's method. The accurate evaluation of derivatives is useful for example, if in the real case the local maxima of $\operatorname{Im} v$ should be determined. - For an asymptotic formula for these maxima see [9], p. 165 or more recently Berryman [2].

\section{Foundations}

Let $y_{1}(x, \lambda), y_{2}(x, \lambda)$ denote the solutions of the differential equation

$$
y^{\prime \prime}(x)+(\lambda+q(x)) y(x)=0, \quad q(x):=\sum_{\kappa=1}^{k}\left(2 t_{\kappa}\right) \cos (2 \kappa x)
$$

related to the initial conditions

$$
\left(\begin{array}{l}
y_{1}(0, \lambda) \\
y_{1}^{\prime}(0, \lambda)
\end{array}\right)=\left(\begin{array}{l}
1 \\
0
\end{array}\right)=: e_{1}, \quad\left(\begin{array}{l}
y_{2}(0, \lambda) \\
y_{2}^{\prime}(0, \lambda)
\end{array}\right)=\left(\begin{array}{l}
0 \\
1
\end{array}\right)=: e_{2} .
$$

As is well known, $v \in \mathbb{C}$ is a characteristic exponent of (1.1), if and only if one of the following mutually equivalent equations holds

$$
\begin{aligned}
& \sin ^{2}\left(\frac{\pi}{2} v\right)=-y_{1}^{\prime}\left(\frac{\pi}{2}, \lambda\right) y_{2}\left(\frac{\pi}{2}, \lambda\right), \\
& \cos ^{2}\left(\frac{\pi}{2} v\right)=y_{1}\left(\frac{\pi}{2}, \lambda\right) y_{2}^{\prime}\left(\frac{\pi}{2}, \lambda\right) .
\end{aligned}
$$

As in [23], Theorem (1.6), we define the following one-sided infinite matrices, in which we set $t_{0}=0, t_{n}=0(n \geqq k+1), t_{-n}=t_{n}(n \in \mathbb{N})$ : 


$$
\begin{aligned}
& C^{(0)}(\lambda)=\left(c_{n, m}^{(0)}(\lambda)\right)_{n, m=0}^{\infty} \\
& c_{n, m}^{(0)}(\lambda):= \begin{cases}\delta_{0, m} \lambda+2 t_{m} & (n=0, m \in \mathbb{N}), \\
\delta_{n, m}\left(1-\frac{\lambda}{4 n^{2}}\right)-\frac{t_{n-m}+t_{n+m}}{4 n^{2}\left(1+\delta_{m, 0}\right)} \quad(n \neq 0, m \in \mathbb{N}),\end{cases} \\
& S^{(0)}(\lambda)=\left(\delta_{n, m}\left(1-\frac{\lambda}{4 n^{2}}\right)-\frac{t_{n-m}-t_{n+m}}{4 n^{2}}\right)_{n, m=1}^{\infty}, \\
& C^{(1)}(\lambda)=\left(\delta_{n, m}\left(1-\frac{\lambda}{(2 n+1)^{2}}\right)-\frac{t_{n-m}+t_{n+m+1}}{(2 n+1)^{2}}\right)_{n, m=0}^{\infty}, \\
& S^{(1)}(\lambda)=\left(\delta_{n, m}\left(1-\frac{\lambda}{(2 n+1)^{2}}\right)-\frac{t_{n-m}-t_{n+m+1}}{(2 n+1)^{2}}\right)_{n, m=0}^{\infty} .
\end{aligned}
$$

These matrices are of "Hill's type" in the sense of [23], (1), (1'). Magnus ([7], Theorem 2.10) has proved the relations

$$
\begin{array}{ll}
y_{1}^{\prime}\left(\frac{\pi}{2}, \lambda\right)=-\frac{\pi}{2} \operatorname{det} C^{(0)}(\lambda), & y_{2}\left(\frac{\pi}{2}, \lambda\right)=\frac{\pi}{2} \operatorname{det} S^{(0)}(\lambda) \\
y_{1}\left(\frac{\pi}{2}, \lambda\right)=\operatorname{det} C^{(1)}(\lambda), & y_{2}^{\prime}\left(\frac{\pi}{2}, \lambda\right)=\operatorname{det} S^{(1)}(\lambda)
\end{array}
$$

Using the notations

$$
\begin{array}{ll}
G_{0,1}(\lambda):=y_{1}^{\prime}\left(\frac{\pi}{2}, \lambda\right), & G_{0,2}(\lambda):=y_{2}\left(\frac{\pi}{2}, \lambda\right), \\
G_{1,1}(\lambda):=y_{1}\left(\frac{\pi}{2}, \lambda\right), & G_{1,2}(\lambda):=y_{2}^{\prime}\left(\frac{\pi}{2}, \lambda\right)
\end{array}
$$

we define for given $v \in \mathbb{C}$

$$
G(\lambda):=\sin ^{2}\left(\frac{\pi}{2} v\right)+G_{0,1}(\lambda) G_{0,2}(\lambda)=\cos ^{2}\left(\frac{\pi}{2} v\right)-G_{1,1}(\lambda) G_{1,2}(\lambda)
$$

In this way the given eigenvalue problem is reduced to the problem of finding the zeros of $G$, which is an entire analytic function. In the case $v=0$ or 1 , which is characterized by the existence of $\pi$-periodic or $2 \pi$-periodic solutions of (1.1), only the zeros of the $G_{v, j}$ from (1.6) have to be found. Even periodic solutions of (1.1) exist if $j=1$, odd periodic solutions if $j=2$.

For evaluating a zero of $G$ from (1.7) or $G=G_{v, j}$ from (1.6) we use Newton's method

$$
\lambda^{(i+1)}=\lambda^{(i)}-G^{\prime}\left(\lambda^{(i)}\right)^{-1} G\left(\lambda^{(i)}\right) \quad(i=0,1,2, \ldots),
$$

which starts with a sufficiently close approximation $\lambda^{(0)}$ of the zero $\lambda$ being sought. As $G$ is holomorphic, Newton's method is always locally convergent. It is quadratically convergent if $G^{\prime}(\lambda) \neq 0$. The latter condition is always fulfilled in the important case $G=G_{v, j}$, the parameters $t_{\kappa}$ being real: this can be verified by means of the Eqs. (2.15) in [8], p. 17. 
In the following we give an a posteriori error bound for a value $\lambda^{(i+1)}$ $(i \in \mathbb{N})$, obtained by numerical realization of (1.8). For this we use the notations

$$
\begin{aligned}
& \lambda_{0}:=\lambda^{(i)}, \\
& \lambda_{1}:=\lambda_{0}-G^{\prime}\left(\lambda_{0}\right)^{-1} G\left(\lambda_{0}\right), \\
& \lambda_{1}:=\lambda^{(i+1)}=\mathbf{f l}\left(\lambda_{0}-\tilde{G}^{\prime}\left(\lambda_{0}\right)^{-1} \tilde{G}\left(\lambda_{0}\right)\right) .
\end{aligned}
$$

In the formula for $\tilde{\lambda}_{1}$ we use the approximate values $\tilde{G}\left(\lambda_{0}\right), \tilde{G}^{\prime}\left(\lambda_{0}\right)$, obtained by practical computation. Furthermore we notice that the operations are performed in a floating-point arithmetic. Let some constants $f_{0}, f_{1}$ with

$$
\left|\tilde{G}\left(\lambda_{0}\right)-G\left(\lambda_{0}\right)\right| \leqq f_{0}, \quad\left|\tilde{G}^{\prime}\left(\lambda_{0}\right)-G^{\prime}\left(\lambda_{0}\right)\right| \leqq f_{1}
$$

and

$$
f_{1}<\left|\tilde{G}^{\prime}\left(\lambda_{0}\right)\right|
$$

be known. We then put

$$
\gamma:=\frac{\left|\tilde{G}\left(\lambda_{0}\right)\right|+f_{0}}{\left|\tilde{G}^{\prime}\left(\lambda_{0}\right)\right|-f_{1}}
$$

and assume that with some $C>0$

$$
\left|G^{\prime \prime}(\omega)\right| \leqq C \quad \text { for } \omega \in K\left(\lambda_{0}, 2 \gamma\right):=\left\{\omega \in \mathbb{C}:\left|\omega-\lambda_{0}\right| \leqq 2 \gamma\right\} .
$$

Then we have the

Theorem. If

$$
\eta:=C \gamma \frac{1}{\left|\tilde{G}^{\prime}\left(\lambda_{0}\right)\right|-f_{1}} \leqq \frac{1}{2},
$$

then $G$ in $K\left(\lambda_{0}, 2 \gamma\right)$ has exactly one zero $\lambda$. Setting

$$
\tilde{q}:=\frac{1}{2} \frac{\eta}{1-\eta},
$$

we obtain the estimation

$$
\left|\lambda_{1}-\lambda\right| \leqq \frac{1}{1-\tilde{q}} \gamma \eta
$$

For the proof, we make use of [12], Theorem (6.7.3) concerning Newton's method. For the quantities given there we verify

$$
\rho \leqq \gamma, \kappa \leqq C, h \leqq \eta, q \leqq \tilde{q},
$$

successively, therefore in $K\left(\lambda_{0}, 2 \rho\right)$ lies exactly one zero $\lambda$ of $G$, and from [12], (6.7.6) the assertion (1.15) follows immediately. The fact that $\lambda$ is the only zero of $G$ in the greater circle $K\left(\lambda_{0}, 2 \gamma\right)$ can be verified by part d) of the proof given in $[12]$, p. 134. 
The estimation of $\left|\tilde{\lambda}_{1}-\lambda_{1}\right|$ that is still required is obtained by an elementary analysis. Under the condition that all quantities are real and that a floating-point arithmetic with the unit roundoff $\tau$ is used, we evaluate the error bound

$$
\left|\tilde{\lambda}_{1}-\lambda_{1}\right| \leqq\left(\left|\tilde{\lambda}_{1}\right|+\frac{\left|\tilde{G}\left(\lambda_{0}\right)\right|}{\left|\tilde{G}^{\prime}\left(\lambda_{0}\right)\right|}\right) \tau+\frac{1}{\left|\tilde{G}^{\prime}\left(\lambda_{0}\right)\right|-f_{1}}\left(f_{0}+f_{1} \frac{\left|\tilde{G}\left(\lambda_{0}\right)\right|}{\left|\tilde{G}^{\prime}\left(\lambda_{0}\right)\right|}\right) .
$$

The error bounds mentioned in (1.10), related to the functions defined by (1.6), will be determined in the Sects. 3 and 4 . Hence the corresponding quantities for a more general $G$ as in (1.7) can obviously be evaluated. Likewise the bound $C$ needed in (1.13) can be deduced from the bounds on the derivatives no. $0,1,2$ of the $G_{v, j}$ : these will be given in (2.13). The right-hand sides of (1.15) and (1.16) are relatively insensitive to the error bound $f_{1}$ of $G^{\prime}\left(\lambda_{0}\right)$ as long as $f_{1}$ remains much smaller than $\left|\tilde{G}^{\prime}\left(\lambda_{0}\right)\right|$. Therefore it may be practical to approximate $G^{\prime}\left(\lambda_{0}\right)$ simply by

$$
\tilde{G}^{\prime}\left(\lambda_{0}\right):=\mathbf{f l}\left\{\frac{1}{h}\left(\tilde{G}\left(\lambda_{0}+h\right)-\tilde{G}\left(\lambda_{0}\right)\right)\right\}
$$

with an appropriate $h>0$.

\section{A Priori Bounds}

Let $\bar{x} \in \mathbb{R}, h>0$ be given as well as $\lambda \in \mathbb{C}, q$ as in (1.1). For estimating the solutions

of

$$
z_{j}(x)=\left(\zeta_{j}(x), \zeta_{j}^{\prime}(x)\right)^{t} \quad(j=1,2)
$$

$$
\left\{\begin{array}{l}
\zeta_{j}^{\prime \prime}(x)=-(\lambda+q(x)) \zeta_{j}(x) \quad(x \in[\bar{x}, \bar{x}+h]=: J), \\
z_{j}(\bar{x})=e_{j}
\end{array}\right.
$$

a priori, we choose, more generally than in [24], p. 35, any $\rho \geqq 0$. Then we define

$$
\begin{aligned}
& L=L(\lambda, \rho):=\left|\lambda-\rho^{2}\right|+\sum_{k=1}^{k}\left|2 t_{\kappa}\right|, \\
& \varphi=\varphi(\rho, h):=\min \left\{\rho h, \frac{\pi}{2}\right\} .
\end{aligned}
$$

Theorem. With respect to the quasi-norm

$$
\|w\|:=\left(\begin{array}{l}
\max \left\{\left|w_{1}(x)\right|: x \in J\right\} \\
\max \left\{\left|w_{2}(x)\right|: x \in J\right\}
\end{array}\right) \quad\left(w(x)=\left(\begin{array}{l}
w_{1}(x) \\
w_{2}(x)
\end{array}\right)\right)
$$

in the space $C_{0}\left(J, \mathbb{C}^{2}\right)$, the estimates

(2.4) $\left(\left\|z_{1}\right\|,\left\|z_{2}\right\|\right) \leqq M:=\left(\begin{array}{cc}\cosh (\sqrt{L} h) & \rho^{-1} \sin \varphi \cosh (\sqrt{L} h) \\ \sqrt{L} \sinh (\sqrt{L} h)+\rho \sin \varphi & 1+\rho^{-1} \sin \varphi \sqrt{L} \sinh (\sqrt{L} h)\end{array}\right)$, 
hold, where if $\rho=0$ we define $\rho^{-1} \sin \varphi:=h$. Moreover if $\rho>0$, then

$$
\left(\left\|z_{1}\right\|,\left\|z_{2}\right\|\right) \leqq \tilde{M}:=\left(\begin{array}{cc}
\exp \left(\rho^{-1} L h\right) & 0 \\
\rho\left\{\exp \left(\rho^{-1} L h\right)-1\right\} & 1
\end{array}\right)\left(\begin{array}{cc}
1 & \rho^{-1} \sin \varphi \\
\rho \sin \varphi & 1
\end{array}\right) .
$$

Proof. As it can be shown by analogy with [24], (2.2), the $z_{j}$ with

$$
h(x):=q(x)+\lambda-\rho^{2}
$$

satisfy the integral equations

$$
\begin{aligned}
z_{j}(x)= & \int_{x}^{x}\left(\begin{array}{cc}
-\frac{1}{\rho} \sin (\rho(x-\xi)) h(\xi) & 0 \\
-\cos (\rho(x-\xi)) h(\xi) & 0
\end{array}\right) z_{j}(\xi) d \xi \\
& +\left(\begin{array}{cc}
\cos (\rho(x-\bar{x})) & \frac{1}{\rho} \sin (\rho(x-\bar{x})) \\
-\rho \sin (\rho(x-\bar{x})) & \cos (\rho(x-\bar{x}))
\end{array}\right) e_{j},
\end{aligned}
$$

which we can write as fixed-point problems

$$
z_{j}=T_{j}\left(z_{j}\right) \quad(j=1,2)
$$

in the space $\mathfrak{R}:=C_{0}\left(J, \mathbb{C}^{2}\right)$. If we set

$$
\delta(w, \tilde{w}):=\|w-\tilde{w}\|, \quad \mathfrak{H}:=\left\{a \in \mathbb{R}^{2}: a \geqq 0\right\},
$$

then obviously $(\mathfrak{R}, \delta)$ is a quasi-metric limes-space in the sense of Schäfke [14], for which the assumptions of the fixed-point theorem [14], p. 112 are fulfilled. Furthermore, since the domain of $T:=T_{1}$ or $T_{2}$ is all of $\Re$, we only have to verify the assumptions [14], (2.7). Let any $w, \tilde{w} \in \mathfrak{R}$ be given; then for $w^{\kappa}$ $:=T^{\kappa}(w), \tilde{w}^{\kappa}:=T^{\kappa}(\tilde{w})(\kappa \in \mathbb{N}, \geqq 1), x \in J$ the inequalities

$$
\begin{aligned}
& \left|w_{1}^{\kappa}(x)-\tilde{w}_{1}^{\kappa}(x)\right| \leqq \frac{L^{\kappa}}{(2 \kappa) !}(x-\bar{x})^{2 \kappa} \max _{x \in J}\left|w_{1}(x)-\tilde{w}_{1}(x)\right| \\
& \left|w_{2}^{\kappa}(x)-\tilde{w}_{2}^{\kappa}(x)\right| \leqq \frac{L^{\kappa}}{(2 \kappa-1) !}(x-\bar{x})^{2 \kappa-1} \max _{x \in J}\left|w_{1}(x)-\tilde{w}_{1}(x)\right|
\end{aligned}
$$

can be proved in the same way as the somewhat cruder inequalities [24], (2.6). From (2.7) we immediately conclude that for $w, \tilde{w} \in \mathfrak{R}, \kappa \geqq 1$

$$
\delta\left(T^{\kappa}(w), \quad T^{\kappa}(\tilde{w})\right) \leqq P_{\kappa} \delta(w, \tilde{w}), \quad P_{\kappa}:=\left(\begin{array}{cc}
\frac{1}{(2 \kappa) !} L^{\kappa} h^{2 \kappa} & 0 \\
\frac{1}{(2 \kappa-1) !} L^{\kappa} h^{2 \kappa-1} & 0
\end{array}\right),
$$

hence the operators $T_{1}$ and $T_{2}$ satisfy all assumptions of the mentioned fixedpoint theorem. The assertion [14], (2.12) applied to $n=1$ yields the estimations

$$
\delta\left(z_{j}, \tilde{z}\right) \leqq\left(I+\sum_{\kappa=1}^{\infty} P_{\kappa}\right) \delta\left(\tilde{z}, T_{j}(\tilde{z})\right)=\left(\begin{array}{cc}
\cosh (\sqrt{L} h) & 0 \\
\sqrt{L} \sinh (\sqrt{L} h) & 1
\end{array}\right) \delta\left(\tilde{z}, T_{j}(\tilde{z})\right)
$$


for the unique fixed points $z_{j}$ of the $T_{j}$, where $\tilde{z} \in \Re$ is arbitrary. Hence we conclude (2.4) by choosing $\tilde{z}=0$ and noticing that because of (2.6)

$$
\left(\left\|T_{1}(0)\right\|,\left\|T_{2}(0)\right\|\right)=\left(\begin{array}{cc}
1 & \rho^{-1} \sin \varphi \\
\rho \sin \varphi & 1
\end{array}\right) .
$$

In the case $\rho>0$ it is possible, for $w, \tilde{w} \in \mathfrak{R}, T=T_{1}$ or $T_{2}, \kappa \geqq 1$ to prove the inequalities

$$
\delta\left(T^{\kappa}(w), T^{\kappa}(\tilde{w})\right) \leqq \tilde{P} \delta(w, \tilde{w}), \quad \tilde{P_{\kappa}}:=\left(\begin{array}{ll}
\frac{1}{\kappa !}\left(\frac{L h}{\rho}\right)^{\kappa} & 0 \\
\frac{\rho}{\kappa !}\left(\frac{L h}{\rho}\right)^{\kappa} & 0
\end{array}\right)
$$

by analogy with $[24],\left(2.6^{\prime}\right)$ : this yields $\left(2.4^{\prime}\right)$.

In the following we confine ourselves to the interval $J=\left[0, \frac{\pi}{2}\right]$. Then the solutions $\zeta_{j}(x)$ of $(2.1)$ are just the $y_{j}(x, \lambda)$ specified in (1.2). If the corresponding $z_{j}(x)$ are named $z_{j}(x, \lambda)$ and if we define

$$
\left[\Phi_{j}(\lambda)\right](x):=z_{j}(x, \lambda) \quad\left(\lambda \in \mathbb{C}, x \in\left[0, \frac{\pi}{2}\right]\right),
$$

then obviously the $\Phi_{j}$ are holomorphic mappings from $\mathbb{C}$ into the Banach space $C_{0}\left([0, \pi / 2], \mathbb{C}^{2}\right)$. For a given $\lambda \in \mathbb{C}$ we represent the $\kappa$-th derivative $\Phi_{j}^{(\kappa)}(\lambda)$ by means of Cauchy's integral formula, integrating around a circle $S\left(\lambda, \alpha_{\kappa}\right)$ with centre $\lambda$ and radius $\alpha_{\kappa}>0$. Let the matrices $M, \tilde{M}$ from (2.4), $\left(2.4^{\prime}\right)$ - with $h=\pi / 2, \rho \geqq 0$ fixed - now be denoted by $M(L), \tilde{M}(L)$. Because of $L(\omega, \rho) \leqq L(\lambda, \rho)$ $+\alpha_{\kappa}$ one has for $\omega \in S\left(\lambda, \alpha_{\kappa}\right)$

$$
\left(\left\|\Phi_{1}(\omega)\right\|,\left\|\Phi_{2}(\omega)\right\|\right) \leqq(\tilde{M})(L(\omega, \rho)) \leqq\left(\tilde{M}\left(L+\alpha_{\kappa}\right),\right.
$$

where again $L=L(\lambda, \rho)$. By this means we obtain estimates for $\left\|\Phi_{j}^{(\kappa)}(\lambda)\right\|$ still containing the parameter $\alpha_{\kappa}$; we choose $\alpha_{\kappa}=\pi^{-1} 2 \rho \kappa$ if we estimate using $\tilde{M}$, otherwise we choose $\alpha_{\kappa}=\kappa$. Obviously, the estimation by means of $M$ is always preferable in the case $0<\rho \leqq 1$. In summary we obtain the

Corollary. Let for $\kappa \in \mathbb{N}, L \geqq 0$

$$
M^{(\kappa)}(L):=
$$

$$
\frac{\kappa !}{\kappa^{\kappa}} \cdot\left(\begin{array}{cc}
\cosh \left(\sqrt{L+\kappa} \frac{\pi}{2}\right) & \rho^{-1} \sin \varphi \cosh \left(\sqrt{L+\kappa} \frac{\pi}{2}\right) \\
\sqrt{L+\kappa} \sinh \left(\sqrt{L+\kappa} \frac{\pi}{2}\right)+\rho \sin \varphi & 1+\rho^{-1} \sin \varphi \sqrt{L+\kappa} \sinh \left(\sqrt{L+\kappa} \frac{\pi}{2}\right)
\end{array}\right)
$$

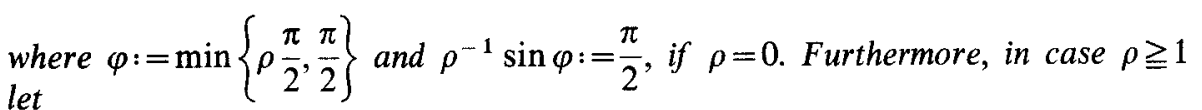

$$
\tilde{M}^{(\kappa)}(L):=\frac{\kappa !}{\kappa^{\kappa}}\left(\frac{\pi}{2 \rho}\right)^{\kappa} \exp \left(\frac{\pi L}{2 \rho}+\kappa\right)\left(\begin{array}{cc}
1 & \rho^{-1} \\
\rho & 1
\end{array}\right)
$$


Then for $\lambda \in \mathbb{C}, \kappa \in \mathbb{N}$, with $L=L(\lambda, \rho)$ from (2.2), there hold the estimates

$$
\left(\left\|\Phi_{1}^{(\kappa)}(\lambda)\right\|,\left\|\Phi_{2}^{(\kappa)}(\lambda)\right\|\right) \leqq \begin{cases}M^{(\kappa)}(L) & \text { in every case, } \\ \tilde{M}^{(\kappa)}(L) & \text { in the case } \rho \geqq 1 .\end{cases}
$$

After the Definition (2.3) of the quasi-norm, the components of $\left[\Phi_{j}^{(\kappa)}(\lambda)\right](x)$ can be estimated by means of (2.12) for any $x \in\left[0, \frac{\pi}{2}\right]$. With a view to the application in Newton's method - cf. (1.13) - we note the

Corollary. Let $\lambda \in \mathbb{C}, \alpha \geqq 0, \kappa \in \mathbb{N}$ be given. Then with any $\rho \geqq 0, L:=L(\lambda, \rho)$ the following estimations are valid for $\omega \in \mathbb{C}$ with $|\omega-\lambda| \leqq \alpha$ :

$$
\left(\begin{array}{ll}
\left|G_{1,1}^{(\kappa)}(\omega)\right| & \left|G_{0,2}^{(\kappa)}(\omega)\right| \\
\left|G_{0,1}^{(\kappa)}(\omega)\right| & G_{1,2}^{(\kappa)}(\omega) \mid
\end{array}\right) \leqq \begin{cases}M^{(\kappa)}(L+\alpha) & \text { in every case, } \\
\tilde{M}^{(\kappa)}(L+\alpha), & \text { if } \rho \geqq 1 .\end{cases}
$$

For the proof we refer to the Definition (1.6) of the $G_{v, j}$ and use $L(\omega, \rho) \leqq L(\lambda, \rho)+\alpha$. - In the practical application of $(2.12),(2.13)$ the choice cf. $[24]-$

$$
\rho=\sqrt{\lambda_{+}}, \quad \lambda_{+}=\max \{\operatorname{Re}(\lambda), 0\}
$$

will be advantageous. In the case $\rho \geqq 1$ one should evaluate both $M^{(\kappa)}$ and $\tilde{M}^{(\kappa)}$ and use the smaller components for estimation.

\section{The Method Based on Numerical Integration}

For given $\lambda \in \mathbb{C}$ the value of $G_{v, j}(\lambda)(v \in\{0,1\}, j \in\{1,2\})$ can be computed by the numerical solution of the differential Eq. (1.1) in the interval $[0, \pi / 2]$, related to the initial condition $z(0)=e_{j}$ : for this we use the Taylor method, on which we have given full details in [24]. In order to compute $G_{v, j}^{\prime}(\lambda)$ for the same $\lambda$, we note that

$$
\left[\Phi_{j}^{\prime}(\lambda)\right](x)=\frac{\partial}{\partial \lambda} z(x, \lambda)=: w(x)=\left(\eta(x), \eta^{\prime}(x)\right)^{t}
$$

satisfies the differential equation

$$
\eta^{\prime \prime}(x)+(\lambda+q(x)) \eta(x)=-y_{j}(x, \lambda) ; \quad \eta(0)=\eta^{\prime}(0)=0 .
$$

Setting for abbreviation

$$
g(x):=-(\lambda+q(x)), \quad y(x):=y_{j}(x, \lambda),
$$

from (3.2) we obtain the following equations for the derivatives of $\eta(x)$ :

$$
\eta^{(\mu)}(x)=\sum_{\kappa=0}^{\mu-2}\left(\begin{array}{c}
\mu-2 \\
\kappa
\end{array}\right) g^{(\mu-2-\kappa)}(x) \eta^{(\kappa)}(x)-y^{(\mu-2)}(x) \quad(\mu=2,3,4, \ldots)
$$


Let $y$ be previously evaluated by the Taylor method of the stepwidth $h=\pi /(2 N)$ and order $p$ : then according to [24], (1.10) we may avail ourselves of the approximate values $u_{n}^{(\mu)}$ for the $y^{(\mu)}\left(x_{n}\right)(\mu=0,1, \ldots, p+1 ; n=0,1, \ldots, N-1)$. Therefore we compute $w(x)$ by the Taylor method of the stepwidth $h$ and order $p$ as follows:

$$
\begin{aligned}
w_{0} & =0, \\
w_{n+1} & =w_{n}+\sum_{\mu=1}^{p} \frac{h^{\mu}}{\mu !} w_{n}^{(\mu)} \quad(n=0,1, \ldots, N-1),
\end{aligned}
$$

where the $w_{n}^{(\mu)}=\left(\eta_{n}^{(\mu)}, \eta_{n}^{(\mu+1)}\right)^{t}$ are determined by $w_{n}=:\left(\eta_{n}, \eta_{n}^{\prime}\right)^{t}$ through the recursion formula

$$
\begin{aligned}
& \eta_{n}^{(0)}=\eta_{n}, \quad \eta_{n}^{(1)}=\eta_{n}^{\prime}, \\
& \eta_{n}^{(\mu)}=\sum_{\kappa=0}^{\mu-2}\left(\begin{array}{c}
\mu-2 \\
\kappa
\end{array}\right) g^{(\mu-2-\kappa)}\left(x_{n}\right) \eta_{n}^{(\kappa)}-u_{n}^{(\mu-2)} \quad(\mu=2,3, \ldots, p+1) .
\end{aligned}
$$

For programming, (3.5) should be reduced to a form similar to [24], (1.13). For saving storage and computing time it is advisable to carry out (3.5) immediately after [24], (1.13), that is, to solve the inhomogeneous differential Eq. (3.2) simultaneously with the homogeneous (1.1).

For the error estimates concerning $z$ we propose two improvements over [24]. The first one refers to the local discretization error. Since by virtue of (2.12) the quasi-norm (2.3) of $z$ can be estimated by

$$
\|z\| \leqq \tilde{M}^{(0)}(L) e_{j}=: K_{j},
$$

and that with $\rho=\sqrt{\lambda_{+}}, L=L(\lambda, \rho)$, in [24], (2.19) we replace $r$ by the sharper bound (depending on $j$ )

$$
r:=\frac{h^{p+1}}{(p+1) !}\left(\begin{array}{ll}
a_{p+1}^{1} & a_{p+1}^{2} \\
a_{p+2}^{1} & a_{p+2}^{2}
\end{array}\right) K_{j} .
$$

The second improvement refers to the matrix $Q$, which according to [24], $\S 4$ characterizes the propagation of errors. For fixed $n \in\{0,1, \ldots, N-1\}$ let $Z(x)$ denote the fundamental matrix of Hill's differential equation related to the initial condition $Z\left(x_{n}\right)=I$, which implies that the columns of $Z(x)$ are $z_{1}(x)$, $z_{2}(x)$ from (2.1) with $J=\left[x_{n}, x_{n+1}\right]$. By virtue of [24], (2.11) we have $Z^{(\mu)}\left(x_{n}\right)$ $=A_{n}^{(\mu)}(\mu \in \mathbb{N})$, therefore we conclude from Taylor's theorem that the matrix which appears in [24], (4.4) can be written as

$$
I+\sum_{\mu=1}^{p} \frac{h^{\mu}}{\mu !} A_{n}^{(\mu)}=Z\left(x_{n+1}\right)-\frac{h^{p+1}}{p !} \int_{0}^{1}(1-t)^{p} Z^{(p+1)}\left(x_{n}+t h\right) d t .
$$

For any matrix $A=\left(a_{i, j}\right)$ we define $|A|:=\left(\left|a_{i, j}\right|\right)$. Then according to [24], (2.10)(2.17) for $x \in\left[x_{n}, x_{n}+h\right]$ we firstly obtain

$$
\left|Z^{(p+1)}(x)\right| \leqq\left(\begin{array}{ll}
a_{p+1}^{1} & a_{p+1}^{2} \\
a_{p+2}^{1} & a_{p+2}^{2}
\end{array}\right)|Z(x)|,
$$


secondly, because of (2.4), we have $|Z(x)| \leqq M$, taken with $\rho=\sqrt{\lambda_{+}}$, and finally

$$
\left|I+\sum_{\mu=1}^{p} \frac{h^{\mu}}{\mu !} A_{n}^{(\mu)}\right| \leqq\left(I+\frac{h^{p+1}}{(p+1) !}\left(\begin{array}{cc}
a_{p+1}^{1} & a_{p+1}^{2} \\
a_{p+2}^{1} & a_{p+2}^{2}
\end{array}\right)\right) M=: Q .
$$

This new $Q$ is more advantageous than the $Q$ from [24], in particular if greater values of $\operatorname{Re}(\lambda)$ are given. Herewith we define

$$
H:=\sum_{\mu=0}^{N-1} Q^{\mu},
$$

and as in [24], (4.7) we obtain the error bound

$$
\left|\tilde{v}_{n}-z\left(x_{n}\right)\right| \leqq H(s+r) \quad(n=0,1, \ldots, N) .
$$

As mentioned in [24], this estimate is rather realistic if the parameters $\lambda, t_{\kappa}$ are not too large. In the following let the $t_{\kappa} \in \mathbb{R}$ be fixed: in view of higher eigenvalues we study the behaviour of $H$ as $\lambda \rightarrow \infty(\lambda>0)$ and $h=\pi /(2 N) \rightarrow 0$, simultaneously. More precisely, setting $\rho=\sqrt{\lambda}$, we suppose

$$
N=O(\rho) \quad(\rho \rightarrow \infty),
$$

and finally, let $p \geqq 3$. Under these assumptions $L=L(\lambda, \rho)$ is independent of $\rho$, the $\varphi(\rho, h)$ are located in an interval $[\psi, \pi / 2]$ with $\psi>0$. Therefore, $Q(\approx M)$ has the asymptotic representation

$$
Q=\left(\begin{array}{cc}
1+O\left(h^{2}\right) & \frac{1}{\rho}\left\{\sin (\varphi(\rho, h))+O\left(h^{2}\right)\right\} \\
\rho\left\{\sin (\varphi(\rho, h))+O\left(h^{2}\right)\right\} & 1+O\left(h^{2}\right)
\end{array}\right),
$$

correspondingly, the eigenvalues of $Q$ are

$$
\mu_{1}=1+\sin (\varphi(\rho, h))+O\left(h^{2}\right), \quad \mu_{2}=1-\sin (\varphi(\rho, h))+O\left(h^{2}\right) .
$$

According to [24], (4.8), we finally have

$$
H=\left(\begin{array}{cc}
\frac{\omega_{1}+\omega_{2}}{2}\left(1+O\left(h^{2}\right)\right) & \frac{\omega_{1}-\omega_{2}}{2} \frac{1}{\rho}\left(1+O\left(h^{2}\right)\right) \\
\rho \frac{\omega_{1}-\omega_{2}}{2}\left(1+O\left(h^{2}\right)\right) & \frac{\omega_{1}+\omega_{2}}{2}\left(1+O\left(h^{2}\right)\right)
\end{array}\right),
$$

with

$$
\omega_{i}=\sum_{\kappa=0}^{N-1} \mu_{i}^{\kappa}=\left(\mu_{i}^{N}-1\right) /\left(\mu_{i}-1\right) \quad(i=1,2)
$$

We discuss the growth of $\omega_{1}$ in two special cases. Firstly, if $N \geqq \rho$ and consequently $\varphi(\rho, h)=\pi / 2$ we have

$$
\omega_{1}=2^{N}\left(1+O\left(N^{-1}\right)\right) \text {. }
$$

Secondly, if $N$ is chosen as $N \approx m \rho$ with a somewhat greater $m \in \mathbb{N}$ we have

$$
\omega_{1} \approx \frac{2 m}{\pi} \exp \left(\frac{\pi}{2} \rho\right)
$$


Obviously, for given $\rho=\sqrt{\lambda}$ the first formula yields a better value. In fact, experience shows that for greater $\lambda$ we can choose $N$ smaller than $\rho$ without requiring too high an order $p$.

The local discretizating error related to $w$ is denoted by the remainder term of the Taylor expansion, hence by

$$
r_{n}^{w}:=\frac{h^{p+1}}{p !} \int_{0}^{1}(1-t)^{p} w^{(p+1)}\left(x_{n}+t h\right) d t .
$$

For its estimation we note that the $\eta^{(\mu)}(x)$ satisfy the linear inhomogeneous recursion (3.3). Consequently, there is a representation

$$
\eta^{(\mu)}(x)=\alpha_{\mu}^{1}(x) \eta(x)+\alpha_{\mu}^{2}(x) \eta^{\prime}(x)+\beta_{\mu}(x),
$$

where the $\alpha_{\mu}^{i}(x)$ are defined by [24], (2.11), the $\beta_{\mu}(x)$ solve the recursion (3.3) with the initial values $\beta_{0}(x)=\beta_{1}(x)=0$. Using the $F_{\mu}$ and $a_{\mu}^{i}$ from [24], (2.14), (2.15) and $K_{j}$ from (3.6), we define

$$
Y_{\mu}:=\left(a_{\mu}^{1}, a_{\mu}^{2}\right) K_{j} \quad(\mu \in \mathbb{N})
$$

and further

$$
b_{0}=b_{1}=0, b_{\mu}=\sum_{\kappa=0}^{\mu-2}\left(\begin{array}{c}
\mu-2 \\
\kappa
\end{array}\right) F_{\mu-2-\kappa} b_{\kappa}+Y_{\mu-2} \quad(\mu=2,3,4, \ldots) .
$$

Because of [24], (2.12) we can estimate $\left|y^{(\mu)}(x)\right| \leqq Y_{\mu}$ and consequently $\left|\beta_{\mu}(x)\right| \leqq b_{\mu}(\mu \in \mathbb{N}, x \in[0, \pi / 2])$. Furthermore, according to (2.12) we have for $x \in[0, \pi / 2]$

$$
|w(x)| \leqq\|w\|=\stackrel{(\tilde{M}}{(1)}^{(1)}(L) e_{j}=: K_{j}^{(1)},
$$

again with $\rho:=\sqrt{\lambda_{+}}$. Therefore, from (3.15) we deduce the estimate

$$
\left|r_{n}^{w}\right| \leqq r^{w}:=\frac{h^{p+1}}{(p+1) !}\left\{\left(\begin{array}{ll}
a_{p+1}^{1} & a_{p+1}^{2} \\
a_{p+2}^{1} & a_{p+2}^{2}
\end{array}\right) K_{j}^{(1)}+\left(\begin{array}{l}
b_{p+1} \\
b_{p+2}
\end{array}\right)\right\} .
$$

Let us sketch the analysis of the local rounding-errors. Let $\tilde{w}_{n}=\left(\tilde{\eta}_{n}, \tilde{\eta}_{n}^{\prime}\right)^{t}$ be the numerical values obtained by the practical realization of the algorithm (3.4), (3.5); let $\tilde{w}_{n}^{(\mu)}=\left(\tilde{\eta}_{n}^{(\mu)}, \tilde{\eta}_{n}^{(\mu+1)}\right)^{t}$ be defined by

$$
\begin{aligned}
& \tilde{\eta}_{n}^{(0)}=\tilde{\eta}_{n}, \quad \tilde{\eta}_{n}^{(1)}=\tilde{\eta}_{n}^{\prime}, \\
& \tilde{\eta}_{n}^{(\mu)}=\sum_{\kappa=0}^{\mu-2}\left(\begin{array}{c}
\mu-2 \\
\kappa
\end{array}\right) g^{(\mu-2-\kappa)}\left(x_{n}\right) \tilde{\eta}_{n}^{(\kappa)}-y^{(\mu-2)}\left(x_{n}\right) \quad(\mu=2,3, \ldots, p+1) .
\end{aligned}
$$

Then the local rounding-error $s_{n}^{w}$ is defined by the equation

$$
\tilde{w}_{n+1}=\tilde{w}_{n}+\sum_{\mu=1}^{p} \frac{h^{\mu}}{\mu !} \tilde{w}_{n}^{(\mu)}+s_{n}^{w} .
$$


In order to find a representation of $s_{n}^{w}$, (3.19) has to be compared with the floating-point-arithmetic version of (3.5). Then the $y^{(\mu-2)}\left(x_{n}\right)$ in (3.19) correspond with the approximating values $\tilde{\tilde{u}}_{n}^{(\mu-2)}$ for the $u_{n}^{(\mu-2)}$. By using [24], (3.4), (3.15), Hilfssatz 3.10, as well as (3.11), we obtain the estimates

$$
\left|y^{(\mu-2)}\left(x_{n}\right)-\tilde{\tilde{u}}_{n}^{(\mu-2)}\right| \leqq\left(a_{\mu-2}^{1}, a_{\mu-2}^{2}\right) H(s+r)+E_{\mu-2} .
$$

The errors which are caused by the arithmetical operations occurring in (3.4), (3.5) are treated in a manner similar to [24], Sect. 3. Explicit formulas for an upper bound $s^{w} \in \mathbb{R}^{2}$ with $\left|s_{n}^{w}\right| \leqq s^{w}(n=0,1, \ldots, N)$ are given in [25].

In order to estimate the global error, we have to compare (3.20) with the Taylor expansion of $w$ in $x_{n}$. Using a procedure similar to [24], Sect. 4 we obtain

$$
\left|\tilde{w}_{n}-w\left(x_{n}\right)\right| \leqq H\left(s^{w}+r^{w}\right) \quad(n=0,1, \ldots, N) .
$$

Since $s^{w}$ contains a linear combination of the right-hand sides of (3.21), the matrix $H$ is included in (3.22) twice. For that reason, in the case of large parameters the presented error analysis will not be practicable.

\section{The Determinantal Method for Mathieu's Differential Equation}

In accordance with the notation of [22] we write Mathieu's differential equation in the form

$$
y^{\prime \prime}(x)+4(\lambda+2 t \cos (2 x)) y(x)=0
$$

consequently the parameter $\lambda$ appearing in (1.1) is now denoted by $4 \lambda$, furthermore one has $k=1, t_{1}=4 t$. Because of (1.5) the functions from (1.6) are equal to

$$
-\frac{\pi}{2} \operatorname{det} C^{(0)}(4 \lambda), \frac{\pi}{2} \operatorname{det} S^{(0)}(4 \lambda), \operatorname{det} C^{(1)}(4 \lambda), \operatorname{det} S^{(1)}(4 \lambda),
$$

successively, where the matrices defined by (1.4) now have tridiagonal form. For getting a common index set for the matrix coefficients, we supply one row and one column with subscript 0 in $S^{(0)}(4 \lambda)$ by

$$
s_{0, n}^{(0)}(4 \lambda)=s_{n, 0}^{(0)}(4 \lambda):=\delta_{0, n} \quad(n \in \mathbb{N}) .
$$

If $\mu \in\{0,1\}$ is given, for $n \in \mathbb{N}$ we write briefly

$$
n^{\prime}:=n+\frac{\mu}{2}
$$

with this we define

$$
1-\eta_{n}^{(\mu)}(\lambda):=1-\frac{\lambda}{n^{\prime 2}}(n \geqq 1-\mu), \quad 1-\eta_{0}^{(0)}(\lambda):=1 .
$$

Then

$$
\tilde{C}^{(\mu)}(\lambda):=\left(\frac{c_{n, m}^{(\mu)}(4 \lambda)}{1-\eta_{n}^{(\mu)}(\lambda)}\right)_{n, m=0}^{\infty}, \quad \tilde{S}^{(\mu)}(\lambda):=\left(\frac{s_{n, m}^{(\mu)}(4 \lambda)}{1-\eta_{n}^{(\mu)}(\lambda)}\right)_{n, m=0}^{\infty}
$$


are almost identical with the matrices, which in [22], (5), (6) are denoted by $C^{(\mu)}, S^{(\mu)}$ respectively, merely the row no. 0 of $\tilde{C}^{(0)}(\lambda)$ is multiplicated by $4 \lambda$ here. In the following let

$$
\tilde{A}(\lambda)=\left(\tilde{a}_{n, m}(\lambda)\right)_{n, m=0}^{\infty}
$$

denote one of the matrices $\tilde{C}^{(\mu)}(\lambda)$ or $\tilde{S}^{(\mu)}(\lambda)$ from (4.3), let the corresponding matrix $C^{(\mu)}(4 \lambda)$ or $S^{(\mu)}(4 \lambda)$ be named

$$
A(\lambda)=\left(a_{n, m}(\lambda)\right)_{n, m=0}^{\infty} .
$$

Furthermore we omit the superscript $\mu$ in $\eta_{n}^{(\mu)}(\lambda)$, which is now fixed. We adopt the $1-\beta_{n}(\lambda):=1-\beta_{n}$ from [22], Satz 1, we define

$$
1-\xi_{n}(\lambda):=\left(1-\eta_{n}(\lambda)\right)\left(1-\beta_{n}(\lambda)\right) \quad(n \in \mathbb{N})
$$

and put

$$
B(\lambda)=\left(b_{n, m}(\lambda)\right)_{n, m=0}^{\infty}:=\left(\frac{\tilde{a}_{n, m}(\lambda)}{1-\beta_{n}(\lambda)}\right)_{n, m=0}^{\infty}=\left(\frac{a_{n, m}(\lambda)}{1-\xi_{n}(\lambda)}\right)_{n, m=0}^{\infty} .
$$

Obviously $B(\lambda)$ is identical with the matrix $B$ from [22]. The numerical evaluation of $\operatorname{det} A(\lambda)$ is based on the relation

$$
\operatorname{det} A(\lambda)=\prod_{n=0}^{\infty}\left(1-\xi_{n}(\lambda)\right) \operatorname{det} B(\lambda) .
$$

For the infinite product of the $1-\beta_{n}(\lambda)$ an explicit formula is given in [22], Satz 2. Furthermore one has

$$
\prod_{n=0}^{\infty}\left(1-\eta_{n}(\lambda)\right)= \begin{cases}(\pi \sqrt{\lambda})^{-1} \sin (\pi \sqrt{\lambda}) & (\mu=0), \\ \cos (\pi \sqrt{\lambda}) & (\mu=1) .\end{cases}
$$

Contrary to [22], (7), we allow $\lambda$ to be equal or approximately equal to a $N^{\prime 2}(N \in \mathbb{N})$. In this case we replace $1-\eta_{N}(\lambda)$ by 1 , and in $1-\tilde{\beta}_{N, 1}^{(\mu)}, 1-\tilde{\beta}_{N+1,2}^{(\mu)}$ from [22], (15) we replace the divisors $N^{\prime 2}-\lambda$ by $N^{\prime 2}$; in an analogous way we treat $1-\widetilde{\beta}_{N+1,2}^{(\mu)}$ in the case $N^{\prime 2} \approx \lambda+1$. The infinite products of the modified $1-\eta_{n}(\lambda), 1-\widetilde{\beta}_{n, \kappa}^{(2)}$ can easily be written down by means of the formulas [22], (24). Further modifications of the $1-\beta_{n}(\lambda)$, concerning the second and third terms of the factorization given in [22], (16), have already been discussed in [22].

According to [22], Satz 1 the determinants of the section matrices

$$
B_{N}(\lambda):=\left(b_{n, m}(\lambda)\right)_{n, m=0}^{N},
$$

$\lambda$ being fixed for the present, converge to $\operatorname{det} B(\lambda)$ like a series with terms $O\left(N^{-12}\right)$. Because of (4.6) we approximate $\operatorname{det} A(\lambda)$ by

$$
a_{N}(\lambda):=\prod_{n=0}^{\infty}\left(1-\xi_{n}(\lambda)\right) \operatorname{det} B_{N}(\lambda)
$$

with $N$ sufficiently large. Concerning this, we state the 
(4.9) Theorem. For any bounded domain $\left(\mathfrak{G} \in \mathbb{C}\right.$ there exists $N_{0} \in \mathbb{N}$ such that all $a_{N}(\lambda)$ with $N \geqq N_{0}$ are holomorphic in $\mathfrak{G}$ and converge to $\operatorname{det} A(\lambda)$ uniformly. $\lambda \in \mathbb{6}$

For proving the first part of the theorem we choose $N_{0}$ such that for any

$$
N_{0} \geqq 4+\sqrt{(\lambda+1)_{+}}+2 \sqrt{|t|},
$$

$(\lambda+1)_{+}$being defined according to (2.14). Then for $\lambda \in\left(5, n \geqq N_{0}\right.$ the $1-\xi_{n}(\lambda)$ are given by the unmodified formulas, therefore they are holomorphic in $\mathfrak{G}$. From the relation

$$
a_{N}(\lambda)=\prod_{n=N+1}^{\infty}\left(1-\xi_{n}(\lambda)\right) \operatorname{det} A_{N}(\lambda),
$$

since the product is uniformly convergent for $\lambda \in \mathbb{6}$, it follows that the $a_{N}$ for $N \geqq N_{0}$ are holomorphic in $\left(\mathfrak{5}\right.$. The uniform convergence of the $a_{N}(\lambda)$ will be shown below, following (4.32).

According to this theorem the $a_{N}^{\prime}(\lambda)$ converge to $\frac{d}{d \lambda} \operatorname{det} A(\lambda)$ uniformly in any compact subset of $\mathbb{C}$. A corresponding error estimation will be given below. First we treat the numerical evaluation of $a_{N}^{\prime}(\lambda)$ for given $\lambda \in \mathbb{C}$. By using the abbreviations

$$
\pi_{N}(\lambda):=\prod_{n=N}^{\infty}\left(1-\xi_{n}(\lambda)\right) \quad(\lambda \in \mathbb{C}, N \in \mathbb{N})
$$

differentiation of (4.11) yields the equation

$$
a_{N}^{\prime}(\lambda)=\pi_{N+1}(\lambda)^{-1} \pi_{N+1}^{\prime}(\lambda) a_{N}(\lambda)+\pi_{0}(\lambda) \prod_{\nu=0}^{N}\left(1-\xi_{v}(\lambda)\right)^{-1} \frac{d}{d \lambda} \operatorname{det} A_{N}(\lambda) .
$$

To deal with the latter term first, we define for $n \in \mathbb{N}-$ with $\lambda$ fixed -

$$
\begin{array}{ll}
z_{n}:=\operatorname{det} A_{n}(\lambda), & u_{n}:=\operatorname{det} B_{n}(\lambda)=\prod_{v=0}^{n}\left(1-\xi_{v}(\lambda)\right)^{-1} z_{n}, \\
w_{n}:=\frac{d}{d \lambda} \operatorname{det} A_{n}(\lambda), & x_{n}:=\prod_{v=0}^{n}\left(1-\xi_{v}(\lambda)\right)^{-1} w_{n} .
\end{array}
$$

For $n=0$ and 1 these quantities are evaluated explicitly according to the particular definition of $A(\lambda)$. The further terms are calculable recursively. From

$$
z_{n+1}=\left(1-\frac{\lambda}{\left(n^{\prime}+1\right)^{2}}\right) z_{n}-\frac{t^{2}}{n^{\prime 2}\left(n^{\prime}+1\right)^{2}} z_{n-1} \quad(n=1,2,3, \ldots)
$$

follows the recursion formula for the $u_{n}$

$$
\begin{gathered}
u_{n+1}=\left(1-\xi_{n+1}\right)^{-1}\left\{\left(1-\frac{\lambda}{\left(n^{\prime}+1\right)^{2}}\right) u_{n}-\left(1-\xi_{n}\right)^{-1} \frac{t^{2}}{n^{\prime 2}\left(n^{\prime}+1\right)^{2}} u_{n-1}\right\} \\
(n=1,2,3, \ldots)
\end{gathered}
$$


In order to avoid unnecessary operations in the practical evaluation of (4.15), we define the quantities

$$
\begin{array}{ll}
\varphi_{n}:=\left\{\begin{array}{ll}
1-\lambda / n^{\prime 2} \\
1
\end{array},\right. & \psi_{n}:= \begin{cases}1 / n^{\prime 2}, & \text { if } \eta_{n}=0\left(\text { i.e. } \lambda \approx n^{\prime 2}\right), \\
1 /\left(n^{\prime 2}-\lambda\right), & \text { if } \eta_{n} \neq 0,\end{cases} \\
\kappa_{n}:=\left(1-\beta_{n}\right), & \alpha_{n}:=t \psi_{n} .
\end{array}
$$

With these expressions (4.15) gets the form

$$
u_{n+1}=\kappa_{n+1}^{-1}\left\{\varphi_{n+1} u_{n}-\kappa_{n}^{-1} \alpha_{n} \alpha_{n+1} u_{n-1}\right\} \quad(n=1,2,3, \ldots)
$$

Differentiating (4.14) with respect to $\lambda$ yields a linear inhomogeneous recursion for the $w_{n}$, from which the recursion for the $x_{n}$ can be inferred immediately. This recursion reads, finally,

$$
x_{n+1}=\kappa_{n+1}^{-1}\left\{\varphi_{n+1} x_{n}-\kappa_{n}^{-1} \alpha_{n} \alpha_{n+1} x_{n-1}-\psi_{n} u_{n}\right\} \quad(n=1,2,3, \ldots) .
$$

Since (4.17) contains the same coefficients as (4.16), the $u_{n}$ and $x_{n}$ should be computed simultaneously. Using these quantities, we have according to (4.8),

$$
\left\{\begin{array}{l}
a_{N}(\lambda)=\pi_{0}(\lambda) u_{N} \\
a_{N}^{\prime}(\lambda)=\pi_{N+1}(\lambda)^{-1} \pi_{N+1}^{\prime}(\lambda) a_{N}(\lambda)+\pi_{0}(\lambda) x_{N} .
\end{array}\right.
$$

Whereas we have already discussed the evaluation of $\pi_{0}(\lambda)$, we now turn to the expression

$$
\begin{aligned}
\pi_{N+1}(\lambda)^{-1} \pi_{N+1}^{\prime}(\lambda) & =-\sum_{n=N+1}^{\infty} \frac{\xi_{n}^{\prime}(\lambda)}{1-\xi_{n}(\lambda)} \\
& =\pi_{0}(\lambda)^{-1} \pi_{0}^{\prime}(\lambda)+\sum_{n=1}^{N} \frac{\beta_{n}^{\prime}(\lambda)}{1-\beta_{n}(\lambda)}+\sum_{n=0}^{N} \frac{\eta_{n}^{\prime}(\lambda)}{1-\eta_{n}(\lambda)}
\end{aligned}
$$

For the second equation we require that all $\beta_{n}$ and $\eta_{n}$ are differentiable in $\lambda$. This is obvious as long as $\beta_{n}(\lambda), \eta_{n}(\lambda)$ are given by their original definitions [22], (12) and (4.2) respectively, in particular for all $n \geqq N_{0}$ from (4.10). Otherwise if, for example, an expression $1-\beta_{n}(\lambda)$ is modified so that in the product decomposition by [22], (10), (15), (16) several factors are replaced by 1 , then the analogous definition of $1-\beta_{n}$ has to be used in a whole neighbourhood of $\lambda$. - The finite sums appearing in (4.19) are to be evaluated explicitly. According to [22], Satz 2 and (4.7) the term $\pi_{0}(\lambda)^{-1} \pi_{0}^{\prime}(\lambda)$ is essentially a sum of logarithmic derivatives of functions

$$
\left(\pi \sqrt{\varphi_{\mu}(\lambda)}\right)^{-1} \sin \left(\pi \sqrt{\varphi_{\mu}(\lambda)}\right), \quad \cos \left(\pi \sqrt{\varphi_{\mu}(\lambda)}\right),
$$

perhaps modified as in [22], (24). These logarithmic derivatives can be written down immediately. Some elementary analysis is necessary if the $d_{1}$ given in [22], (14) equals zero, or if - in the case of real parameters - a $d_{\boldsymbol{\kappa}}$ is purely imaginary (cf. [22], p. 58).

The somewhat cumbersome programming of $\pi_{N+1}(\lambda)^{-1} \pi_{N+1}^{\prime}(\lambda)$ can be omitted, if the program should be used only for eigenvalue problems related to 
$v=0$ or 1 . In the case of good starting values in the Newton method, only very small $a_{N}(\lambda)$ occur. Therefore in the expression (4.18) for $a_{N}^{\prime}(\lambda)$ the first term is negligible. An estimation for $\pi_{N+1}(\lambda)^{-1} \pi_{N+1}^{\prime}(\lambda)$ can be determined by means of the first Eq. of (4.19).

Our next aim is an error estimation for $a_{N}(\lambda)$, which, for instance, can be used to complete the proof of Theorem (4.9). For the present let $\lambda \in \mathbb{C}$ be fixed, let the argument $\lambda$ in $A, \xi_{n}$ etc. be omitted for brevity. Let $N_{0}$ with the property (4.10) be chosen. For $N \geqq N_{0}$ we define $\rho_{N}^{(3)}$ and $\gamma_{N}$ according to [11], Satz 5.11 and [11], Satz 6.2 with $l=3$ and $\xi_{N}$ instead of $\beta_{N}$. By analogy with the last equation from [11], p. 46, for $m \geqq N+1$ we derive the following relations

$$
\begin{aligned}
a_{m}-a_{N}= & \sum_{n=N+1}^{m} \pi_{n+1}\left(\gamma_{n} \operatorname{det} A_{n-1}+\rho_{n}^{(3)}\right) \\
= & \sum_{n=N+1}^{m}\left\{\gamma_{n}\left(1-\xi_{n}\right)^{-1} \operatorname{det} A+\pi_{n+1} \rho_{n}^{(3)}\right\} \\
& +\sum_{n=N+1}^{m} \gamma_{n}\left(1-\xi_{n}\right)^{-1}\left\{\pi_{n} \operatorname{det} A_{n-1}-\operatorname{det} A\right\} .
\end{aligned}
$$

Here we substitute $\pi_{n} \operatorname{det} A_{n-1}=a_{n-1}$. We notice that $\operatorname{det} A=\lim _{k \rightarrow \infty} a_{k}$, and in this way for $m \geqq N \geqq N_{0}$ we obtain the inequalities

$$
\begin{gathered}
\left|a_{m}-a_{N}\right| \leqq \\
+\operatorname{det} A\left|\sum_{n=N+1}^{\infty}\right| \gamma_{n}\left(1-\xi_{n}\right)^{-1}\left|+\sum_{n=N+1}^{\infty}\right| \pi_{n+1} \rho_{n}^{(3)} \mid \\
+\sup _{n, k \geqq N}\left|a_{n}-a_{k}\right| \sum_{n=N+1}^{\infty}\left|\gamma_{n}\left(1-\xi_{n}\right)^{-1}\right| .
\end{gathered}
$$

Since the right-hand side is independent of $m$ and monotonically decreasing with respect to $N$, the left side may be replaced by $\sup \left\{\left|a_{m}-a_{n}\right|: n, m \geqq N\right\}$. On condition that

$$
\sum_{n=N+1}^{\infty}\left|\gamma_{n}\left(1-\xi_{n}\right)^{-1}\right| \leqq g_{N}<1 \quad\left(N \geqq N_{1} \geqq N_{0}\right)
$$

we obtain for $N \geqq N_{1}$ the estimates

$$
\begin{aligned}
\left|\operatorname{det} A-a_{N}\right| & \leqq \sup _{n, m \geqq N}\left|a_{n}-a_{m}\right| \\
& \leqq\left(1-g_{N}\right)^{-1}\left\{g_{N}|\operatorname{det} A|+\sum_{n=N+1}^{\infty}\left|\pi_{n+1} \rho_{n}^{(3)}\right|\right\} .
\end{aligned}
$$

In order to determine error bounds by explicit formulas, we can partially refer to the results of [22]. For distinction here we shall mark the submatrices of $\tilde{A}$ with ; analogously let the $\gamma_{n}$ from [22], (13) be denoted by $\tilde{\gamma}_{n}$. From the connection $\gamma_{n}=\left(1-\eta_{n}\right) \tilde{\gamma}_{n}\left(n \geqq N_{0}\right)$ we conclude that

$$
\sum_{n=N+1}^{\infty}\left|\gamma_{n}\left(1-\xi_{n}\right)^{-1}\right|=\sum_{n=N+1}^{\infty}\left|\tilde{\gamma}_{n}\left(1-\beta_{n}\right)^{-1}\right| \leqq \sup _{n \geqq N+1}\left|\left(1-\beta_{n}\right)^{-1}\right| \sum_{n=N+1}^{\infty}\left|\tilde{\gamma}_{n}\right| .
$$


In this way [22], (34) yields for the $g_{N}$ in (4.22) the possible values

$$
\begin{aligned}
g_{N}:= & \left(1-\frac{|t|^{2}}{\left(N^{\prime}-\sqrt{\lambda_{+}}\right)^{4}}\right)^{-1}\left(1-\frac{|t|^{4}}{\left(N^{\prime}-\sqrt{(\lambda+1)_{+}}\right)^{8}}\right)^{-1} \\
& \cdot \frac{4|\lambda||t|^{4}+\left(1+2 \delta_{N}\right)|t|^{6}}{11\left(N^{\prime}-3-\sqrt{\lambda_{+}}\right)^{11}},
\end{aligned}
$$

where $\delta_{N}$ is defined by [22], (33). Because of the tridiagonal form of $A$ the matrix $U_{n}^{(3)}$ appearing in the definition of $\rho_{n}^{(3)}$ is the number

$$
\left(\begin{array}{l}
n-4 \\
n-4
\end{array} \mid A_{n-1}\right)=\operatorname{det} A_{n-5} \operatorname{det} D_{n}^{(3)} .
$$

Therefore a short calculation yields

$$
\begin{aligned}
& \pi_{n+1} \rho_{n}^{(3)}=\prod_{v=n+1}^{\infty}\left(1-\beta_{v}\right) \\
& \left.\cdot \prod_{v=n-4}^{\infty}\left(1-\eta_{v}\right)\left\{\tilde{z}_{n}^{(3) t} \tilde{D}_{n}^{(3)^{-1}} \tilde{L}_{n}^{(3)}\right)\left(\tilde{R}_{n}^{(3)} \tilde{D}_{n}^{(3)^{-1}} \tilde{S}_{n}^{(3)}\right) \operatorname{det} \tilde{D}_{n}^{(3)}\right\} \operatorname{det} A_{n-5} .
\end{aligned}
$$

The expression in braces can be evaluated and estimated after [22], p. 61. The result is

$$
\begin{aligned}
& \sum_{n=N+1}^{\infty}\left|\pi_{n+1} \rho_{n}^{(3)}\right| \\
& \quad \leqq \delta_{N} \frac{|t|^{8}}{15\left(N^{\prime}-4-\sqrt{\lambda_{+}}\right)^{15}} \sup _{n \geqq N+1}\left|\prod_{v=n-4}^{\infty}\left(1-\eta_{v}\right) \prod_{v=n+1}^{\infty}\left(1-\beta_{v}\right) \operatorname{det} A_{n-5}\right| .
\end{aligned}
$$

From (4.23) in connection with (4.25) we first want to derive a priori bounds. From [22], (30), treating the $1-\eta_{n}$ analogously, we conclude

$$
\begin{aligned}
& \sup _{n \geqq N+1}\left|\prod_{v=n-4}^{\infty}\left(1-\eta_{v}\right) \prod_{v=n+1}^{\infty}\left(1-\beta_{v}\right)\right| \\
& \quad \leqq \exp \left(\frac{|\lambda|}{N^{\prime}-4}+\frac{|t|^{2}}{3\left(N^{\prime}-\sqrt{\lambda_{+}}\right)^{3}}+\frac{|t|^{4}}{7\left(N^{\prime}-\sqrt{(\lambda+1)_{+}}\right)^{7}}\right)=: r_{N} .
\end{aligned}
$$

By estimating the Euclidean norms of the rows of $A$ we obtain the following upper bound for $\left|\operatorname{det} A_{n-5}\right|$ :

$$
P:= \begin{cases}s_{0}\left(\pi \sqrt{|\lambda|+\sqrt{2|t|})^{-1} \sinh (\pi \sqrt{|\lambda|+\sqrt{2}|t|)},},\right. & \text { if } A=C^{(0)}(4 \lambda), S^{(0)}(4 \lambda), \\ \cosh (\pi \sqrt{|\lambda|+\sqrt{2}|t|)} & \text { otherwise, }\end{cases}
$$

where $s_{0}=4\left(|\lambda|^{2}+4|t|^{2}\right)^{1 / 2}$ if $A=C^{(0)}(4 \lambda), s_{0}=1$ if $A=S^{(0)}(4 \lambda)$. For det $A$ itself, a sharper bound results from (2.13) by using the relation (1.5), namely

$$
|\operatorname{det} A| \leqq \tilde{m}_{i, j}^{(0)}(L) \text {, }
$$


where $\tilde{m}_{i, j}^{(0)}(L)$ is the corresponding coefficient of $\stackrel{(\sim)}{M}^{(0)}(L)$ with

$$
\rho=4 \sqrt{\lambda_{+}}, \quad L=\left|4 \lambda-\rho^{2}\right|+8|t| .
$$

In the case $\mu=0$, the right-hand side of (4.28) may be multiplicated by $2 / \pi$. In summary, we note the

\section{A Priori Bound}

$$
\begin{aligned}
& \left|\operatorname{det} A-a_{N}\right| \leqq \sup _{n, m \geqq N}\left|a_{n}-a_{m}\right| \\
& \quad \leqq\left(1-g_{N}\right)^{-1}\left\{g_{N}{\stackrel{(\sim)}{m_{i, j}}(0)}_{(L)}+\delta_{N} r_{N} P \frac{|t|^{8}}{15\left(N^{\prime}-4-\sqrt{\lambda_{+}}\right)^{15}}\right\} .
\end{aligned}
$$

Since for $\operatorname{det} A \approx 0$ the bounds $P$ and $\tilde{m}_{i, j}^{(0)}(L)$ are too crude, we give a variant of estimation. For this we observe that

$$
\prod_{v=n-4}^{\infty}\left(1-\eta_{v}\right) \prod_{v=n+1}^{\infty}\left(1-\beta_{v}\right) \operatorname{det} A_{n-5}=\prod_{v=n-4}^{n}\left(1-\beta_{v}\right)^{-1} a_{n-5} .
$$

After estimating the product of the $\left(1-\beta_{v}\right)^{-1}$ by

$$
p_{N}:=\left(1-\frac{|t|^{2}}{\left(N^{\prime}-4-\sqrt{\lambda_{+}}\right)^{4}}\right)^{-4}\left(1-\frac{|t|^{4}}{\left(N^{\prime}-4-\sqrt{(\lambda+1)_{+}}\right)^{8}}\right)^{-4}
$$

from (4.23), (4.25) we immediately obtain the

\section{A Posteriori Bound}

$$
\left|\operatorname{det} A-a_{N}\right| \leqq\left(1-g_{N}\right)^{-1}\left\{g_{N}|\operatorname{det} A|+\delta_{N} p_{N} \frac{|t|^{8}}{15\left(N^{\prime}-4-\sqrt{\lambda_{+}}\right)^{15}} \sup _{n \geqq N-4}\left|a_{n}\right|\right\} .
$$

For application, we estimate

$$
\left\{\begin{array}{l}
|\operatorname{det} A| \leqq\left|a_{N}\right|+\left|\operatorname{det} A-a_{N}\right|, \\
\sup _{n \geqq N-4}\left|a_{n}\right| \leqq \max \left\{\left|a_{n}\right|: N-4 \leqq n \leqq N-1,\left|a_{N}\right|+\sup _{n, m \geqq N}\left|a_{n}-a_{m}\right|\right\}
\end{array}\right.
$$

by means of the known $a_{n}$ and the bounds from (4.30).

By the use of (4.30) the proof of (4.9) can be completed. If a bounded domain $\left(\mathfrak{b} \subset \mathbb{C}\right.$ is given, we again choose $N_{0}$ so that $(4.10)$ holds for any $\lambda \in(\mathfrak{G}$. Obviously, the right-hand side of (4.30), which depends on $\lambda$, tends to zero uniformly for $\lambda \in \mathfrak{G}$.

In order to estimate the error of $a_{N}^{\prime}(\lambda)$ at a fixed $\lambda \in \mathbb{C}$ we choose $N_{0}$ with

$$
N_{0} \geqq 4+\sqrt{(\lambda+2)_{+}}+2 \sqrt{|t|} .
$$

Then for $N \geqq N_{0}$ the $a_{N}$ are holomorphic in the closed disk of radius 1 around $\lambda$. According to Cauchy's integral formula we have for $N \geqq N_{0}$

$$
\left|a_{N}^{\prime}(\lambda)-\frac{d}{d \lambda} \operatorname{det} A(\lambda)\right| \leqq \max \left\{\left|a_{N}(\omega)-\operatorname{det} A(\omega)\right|: \omega \in \mathbb{C},|\omega-\lambda|=1\right\}
$$


We obtain upper bounds $\delta_{N}^{\prime}, P^{\prime}, g_{N}^{\prime}$ and $r_{N}^{\prime}$ for $\delta_{N}(\omega), P(\omega), g_{N}(\omega)$ and $r_{N}(\omega)$ $\left(\omega \in \mathbb{C},|\omega-\lambda|=1, N \geqq N_{0}\right)$ in the following way: in the formulas for $\delta_{N}, P, g_{N}$ and $r_{N}$ we substitute $(\lambda+1)_{+}$for $\lambda_{+},(\lambda+2)_{+}$for $(\lambda+1)_{+},(|\lambda|+1)$ for $|\lambda|$ and, additionally, $\delta_{N}^{\prime}$ for $\delta_{N}$ in (4.24). According to the definition $A(\omega)=C^{(\mu)}(4 \omega)$ or $S^{(\mu)}(4 \omega)$ we conclude from (2.13) that with the corresponding $(i, j)$

$$
\max _{|\omega-\lambda|=1}|\operatorname{det} A(\omega)| \leqq \tilde{(}_{i, j}^{(0)}(L+4)=: m^{\prime}
$$

where $L, \rho$ are given by (4.29). In summary, for all $N \geqq N_{0}$ with $g_{N}^{\prime}<1$ we note the estimation

$$
\begin{aligned}
& \left|a_{N}^{\prime}(\lambda)-\frac{d}{d \lambda} \operatorname{det} A(\lambda)\right| \\
& \leqq\left(1-g_{N}^{\prime}\right)^{-1}\left\{g_{N}^{\prime} m^{\prime}+\delta_{N}^{\prime} r_{N}^{\prime} P^{\prime} \frac{|t|^{8}}{15\left(N^{\prime}-4-\sqrt{(\lambda+1)_{+}}\right)^{15}}\right\} \text {. }
\end{aligned}
$$

Obviously the right-hand side tends to zero like $O\left(N^{-11}\right)$. A sharper a posteriori estimation for $a_{N}^{\prime}(\lambda)$ can be constructed by differentiating the middle expression from (4.20) and by inserting the terms $\gamma_{n}\left(1-\xi_{n}\right)^{-1} \frac{d}{d \lambda} \operatorname{det} A(\lambda)$. For the resulting estimation, Cauchy's integral formula is needed only partially.

The rounding-errors which occur during the numerical realization of (4.16) have been discussed in [22], p. 61/62 with regard to their asymptotic behaviour as $n \rightarrow \infty$. On the other hand, the recursion formula [22], (38) for the $\varepsilon_{n}$ can be replaced by a similar formula which contains the practically computed $\tilde{u}_{n}$ instead of the $u_{n}$. In this way it is possible to calculate upper bounds for the roundoff-errors recursively: a detailed analysis is given in [25].

\section{Numerical Examples}

For employing Newton's method (1.8) we have to know an appropriate starting value $\lambda^{(0)}$. Given small parameters $t_{\kappa}$, for $\lambda^{(0)}$ we can choose the corresponding eigenvalue of (1.1) with $q=0$. This "unperturbed" problem obviously has the eigenvalues

$$
\hat{\lambda}_{n}=(v+2 n)^{2} \quad(n \in \mathbb{Z}) .
$$

If for greater $\left|t_{\kappa}\right|$ and smaller $|n|$ the Newton method starting from $\lambda_{n}$ does not converge, other methods to construct a suitable $\lambda^{(0)}$ must be inserted. One method consists in solving a sequence of neighbouring eigenvalue problems, namely related to

$$
y^{\prime \prime}(x)+\left(\lambda+\alpha_{\kappa} q(x)\right) y(x)=0 \quad(\kappa=0,1, \ldots, K)
$$

with $0=\alpha_{0}<\alpha_{1}<\ldots<\alpha_{K}=1$, by chosing the eigenvalue of the differential equation no. $\kappa$ for the starting value of the Newton method related to equation no. $\kappa+1$. In the cases $v=0$ or 1 a suitable $\lambda^{(0)}$ is obtained much more ef- 
ficiently by the Rayleigh-Ritz method. Related to the zeros of $G_{0,1}$ this method consists in evaluating the eigenvalues of

$$
\hat{C}_{N}^{(0)}=\left(\hat{c}_{n, m}^{(0)}\right)_{n, m=0}^{N}
$$

with

$$
\begin{array}{ll}
\hat{c}_{n, n}^{(0)}=4 n^{2} \quad(0 \leqq n \leqq N), & \hat{c}_{0, n}^{(0)}=\hat{c}_{n, 0}^{(0)}=-\sqrt{2} t_{n} \quad(1 \leqq n \leqq N), \\
\hat{c}_{n, m}^{(0)}=-\left(t_{n-m}+t_{n+m}\right) & (1 \leqq n, m \leqq N ; n \neq m),
\end{array}
$$

where $N \in \mathbb{N}$ is chosen appropriately. Obviously the eigenvalues of $\hat{C}_{N}^{(0)}$ are the zeros of $\operatorname{det} C_{N}^{(0)}(\lambda)$ according to definition (1.4). With regard to the other $G_{v, j}$ one may proceed in an analogous way.

The algorithms presented in this paper were programmed in FORTRAN, they were carried out on the TR 440 of the University of Regensburg, using double-precision arithmetic. This arithmetic, approximately equivalent to a $25-$ digit decimal arithmetic, was chosen for demonstrating the virtue of the methods, when high accuracy is demanded. In the following examples we restrict ourselves to the zeros of the functions $G=G_{v, j}$ from (1.6), that is to the cases $\nu=0$ or 1 ; the programs are also applicable to a more general $v$.

In the Examples 1 and 2 we present numerical results for the finite Hill's differential Eq. (1.1) with $k>1$, treated by the Taylor method of Sect. 3. In Example 1 the starting values of Newton's method are chosen

$$
\lambda^{(0)}=-\sum_{\kappa=1}^{k}\left|t_{\kappa}\right|
$$

for evaluating the smallest eigenvalue,

$$
\lambda^{(0)}=4 n^{2}+\frac{c}{4 n^{2}}, \quad c=\frac{1}{\pi} \int_{0}^{\pi} q(x)^{2} d x \quad(n=1,2,3, \ldots)
$$

for evaluating the other eigenvalues. In Example 2 a preprocessing calculation is needed for the smaller eigenvalues; we used the differential Eqs. (5.2) with $\alpha_{\kappa}=\kappa \cdot 0.2(\kappa=0,1, \ldots, 5)$. Since for $\alpha_{\kappa}<1$ the eigenvalues need not be computed very accurately, here we stop the Newton iteration as soon as

$$
\left|\lambda^{(i)}-\lambda^{(i+1)}\right|<10^{-2} \max \left\{1,\left|\lambda^{(i)}\right|\right\} .
$$

For the given differential equation $\left(\alpha_{\kappa}=1\right)$ the Newton method is carried out, until

$$
\left|\lambda^{(i)}-\lambda^{(i-1)}\right|<10^{-10} \max \left\{1,\left|\lambda^{(i)}\right|\right\} .
$$

Then a final Newton iteration step follows, in which the Taylor method is applied in conjunction with the error analysis. In all examples the value

$$
N=\min \left\{100,\left[4\left(|\lambda|+\sum_{\kappa=1}^{k}\left|t_{\kappa}\right|^{2}+k^{2}\right)^{\frac{1}{2}}\right]\right\}
$$


turns out to be an appropriate number of subintervals, cf. (3.12). In order to obtain a small bound for the discretization error, the order $p$ is chosen so that for $r$ from (3.7)

$$
r \leqq \tau\left\|K_{j}\right\|_{\infty}^{-1} K_{j} \quad\left(\tau=0.5 \cdot 10^{-24}\right)
$$

is fulfilled. The error estimation for $\lambda^{(i+1)}$ is executed according to (1.15), (1.16), if possible, where the $f_{0}, f_{1}$ are calculated by means of (3.11), (3.22).

Starting from $\lambda^{(i)}$, we also perform a Newton iteration step employing the determinantal method from [23], for comparison. As in [23], (3.11) the stopping criterion

$$
\left|\operatorname{det} B_{N}-\operatorname{det} B_{N-1}\right|<\tilde{\varepsilon} \max \left\{\left|\operatorname{det} B_{N}\right|, 10^{-10}\right\}
$$

with $\tilde{\varepsilon}=10^{-20}$ is employed. The derivative of $\operatorname{det} A(\lambda)$ is approximated by an expression (1.17) with $h=2 \sqrt{\tilde{\varepsilon} C}$, where $C$ is a bound for the second derivative obtained by (2.13).

Example 1. $k=10, t_{\kappa}=\frac{1}{\kappa^{2}}(\kappa=1,2, \ldots, 10), G=G_{0,1}$

$\begin{array}{llll}\begin{array}{l}\text { Eigenvalue } \\ \text { number }\end{array} & \text { Eigenvalue } & \begin{array}{l}\text { Iteration } \\ \text { steps }\end{array} & \begin{array}{l}\text { Error } \\ \text { bound }\end{array} \\ 1 & -0.5055349475163051455684196 & 7 & 5.0 E-22 \\ 3 & \underline{15.98818824438994579243448} & 4 & 4.2 E-20 \\ 6 & \underline{99.99636855162359068394286} & 4 & 1.7 E-16\end{array}$

Example 2. $k=3, t_{1}=-10.0, t_{2}=-3.8809, t_{3}=2.74, G=G_{0,2}$

$\begin{array}{llll}\begin{array}{l}\text { Eigenvalue } \\ \text { number }\end{array} & \text { Eigenvalue } & \begin{array}{l}\text { Iteration } \\ \text { steps }\end{array} & \begin{array}{l}\text { Error } \\ \text { bound }\end{array} \\ 1 & -\underline{7.270108805205036748240416} & 22 & 1.0 E-18 \\ 2 & \underline{16.71664311874277922130561} & 12 & 3.5 E-18 \\ 5 & \underline{100.6387854361530586418444} & 8 & 2.3 E-15\end{array}$

In the tabulated eigenvalues the underlined digits have been confirmed by the determinantal method. In the following column the number of Newtoniteration steps are listed; in the second example the preceding calculations are included. The error bounds were evaluated from (1.15), (1.16) using the error analysis - including the rounding-errors - discussed in Sect.3. For higher eigenvalues $(\lambda>400)$ this error analysis was no longer practicable, since (1.11) or (1.14) were violated. Nevertheless, even in these cases the eigenvalues obtained by the two different methods coincided in more than 20 digits. - The following computation times were needed for the final iteration step:

Taylor method including error analysis

Example $1 \quad$ Example 2

Determinantal method

$\begin{array}{ll}2.5-6.0 \mathrm{~s} & 3.2-6.6 \mathrm{~s} \\ 14-22 \mathrm{~s} & 0.6-1.6 \mathrm{~s}\end{array}$


As mentioned in [23], the computational cost of the determinantal method is proportional to $k^{2}$, the cost of the Taylor method is nearly independent of $k$ : this explains the difference between the Examples 1 and 2.

For several values of $t_{1}$, lower and upper bounds for the leading eigenvalues of Mathieu's differential equation

$$
y^{\prime \prime}(x)+\left(\lambda+2 t_{1} \cos (2 x)\right) y(x)=0
$$

in the even $\pi$-periodical case are tabulated in [26] - see also [4], p. 40 -. According to a differing notation of Mathieu's equation in [26], these eigenvalues are $\lambda+2 t_{1}$, with $\lambda$ the zeros of $G_{0,1}$. The author has treated these examples by the methods of the present paper. For initial approximations we chose the eigenvalues of $\hat{C}_{N}^{(0)}$ from (5.3), computed by means of the $Q L$-method, setling $N=20$ or $N=100$ (in the below example). Starting from these values, we performed only one Newton-iteration step, using each the Taylor method from Sect. 3 and the determinantal method from Sect. 4. For both methods the error analysis including the roundoff-errors was carried out. In order to be able to perform the error analysis of the determinantal method, we determined a minimum number $N$ so that $g_{N}^{\prime}<1$ and the right-hand side of (4.36) became smaller than an $\varepsilon^{\prime}>0$. In the below example we chose $\varepsilon^{\prime}=\sqrt[4]{\tilde{\varepsilon}}$, where

$$
\tilde{\varepsilon}=\tau \max \left\{1,\left|\lambda_{0}\right|\right\}
$$

Additionally we employed the stopping condition (5.5). On the other hand we had limited $N$ by 1,000 .

Here we present only a few of the eigenvalues related to the largest $t_{1}$ tabulated in [26]. Additional, more comprehensive tables are contained in [25].

Example 3. $k=1, t_{1}=250.0, G=G_{0,1}$

Eigenvalue Eigenvalue Error bound

number

$\begin{array}{rcr}1 & -\frac{468.6292484101606399447285}{226.0584032007784231762483} & 1.2 E-19 \\ 3 & -\underline{1.662682553528771920682963} & 1.8 E-17 \\ 5 & \underline{201.5668560274913563409032} & 7.3 E-17 \\ 7 & \underline{450.9847221384010694359174} & 4.0 E-18\end{array}$

The error bounds are derived from the error analysis given in Sect. 4, including the roundoff-errors. The error estimates for the Taylor method are so bad that (1.11) or (1.14) are violated. On the other hand the eigenvalues computed by the Taylor method coincide with the tabulated values in the underlined digits. For all examples the computational times needed for the determinantal method were considerably shorter than for the Taylor method: therefore the determinantal method is always preferable in the case of Mathieu's equation. 
The last example shows that our methods are also appropriate in the case of large parameters. We would like to point out that in [26], p. 220 the eigenvalues of our Example 3 are given only to five decimal places, whereas for the preceding examples in [26] the eigenvalues are accurate to 7 or 8 decimal places.

Finally, the author has translated the ALGOL-program for Jansen and Jeuken's method [6], supplied by Thurlings [20], into FORTRAN. By means of this program, using double precision arithmetic, the examples mentioned for Mathieu's equation were treated. The eigenvalues obtained for Examples 3 coincide with the tabulated values, at least in the underlined decimal digits. The computational times of this method are comparable with those of the determinantal method. If, however, in the determinantal method the error analysis is omitted and, instead of this, stopping conditions like (5.5) also related to the derivatives are employed, the computational times are considerably reduced.

\section{References}

1. Arscott, F.M., Lacroix, R., Shymanski, W.T.: A three term recursion and the computation of Mathieu functions. Proc. Eighth Manitoba Conf. Numer. Math. Comput. pp. 107-115, 1978

2. Berryman, J.G.: Floquet Exponent for Instability Intervals of Hill's Equation. Commun. Pure Appl. Math. 32, 113-120 (1979)

3. Bouwkamp. C.J.: Theoretische en numerische behandeling von de buiging door en ronde opening. Diss. Groningen. Groningen-Batavia, 1941

4. Fichera, G.: Numerical and Quantitative Analysis. London, San Francisco, Melbourne: Pitman, 1978

5. Jansen, J.K.M.: Simple-periodic and non-periodic Lamé functions and their application in the theory of conical waveguide. Diss. Amsterdam, 1976

6. Jansen, J.K.M., Jeuken, M.E.J.: Propagation and radiation properties of elliptical waveguide with anisotropic boundary. Report Dept. of Electrical Engineering, Eindhoven University of Technology, 1973

7. Magnus, W.: Infinite determinants associated with Hill's equation. Pacific J. Math. 5, Suppl. 2, 941-951 (1955)

8. Magnus, W., Winkler, S.: Hill's Equation. New York, London, Sidney: Wiley, 1966

9. Meixner, J., Schäfke, F.W.: Mathieusche Funktionen und Sphäroid-Funktionen. Berlin, Heidelberg, New York: Springer, 1954

10. Mennicken, R.: On the convergence of Infinite Hill-Type Determinants. Arch. Rat. Mech. Anal. 30, 12-37 (1968)

11. Mennicken, R., Wagenführer, E.: Über die Konvergenz verallgemeinerter Hillscher Determinanten. Math. Nachr. 72, 21-49 (1976)

12. Mennicken, R., Wagenführer, E.: Numerische Mathematik II. Reinbek: rororo-Vieweg, 1977

13. Pedersen, P.: Stability of the solutions to Mathieu-Hill equations with damping. Danish Center for Applied Math. and Mech., Report no. 158, 1979

14. Schäfke, F.W.: Zum Anwendungsbereich einiger Iterationsverfahren. Numer. Math. 15, 109-121 (1970)

15. Schäfke, F.W., Schmidt, D.: Ein Verfahren zur Berechnung des charakteristischen Exponenten der Mathieuschen Differentialgleichung, III. Numer. Math. 8, 68-71 (1966)

16. Schröder, J.: Das Iterationsverfahren bei allgemeinerem Abstandsbegriff. Math. Z. 66, 111-116 (1956)

17. Stoer, J., Bulirsch, R.: Einführung in die Numerische Mathematik II, 2. Auflage. Berlin, Heidelberg, New York: Springer, 1978

18. Tamir, T.: Characteristic exponents of Mathieu functions. Math. Comput. 16, 100-106 (1962) 
19. The Group "Numerical Analysis" at Delft University of Technology: On the computation of Mathieu functions. J. Engrg. Math. 7, 39-61 (1973)

20. Thurlings, L.F.G.: Elliptical waveguide as a feed for circularly polarised waves. Report Dept. of Electrical Engineering, Eindhoven University of Technology, 1975

21. van der Krogt, P.A.M.: Eigenwaarden von een klasse von oneindige tridiagonaal matrices. Afstudeerverslag Techn. Hogeschool to Eindhoven, 1968

22. Wagenführer, E.: Ein Verfahren höherer Konvergenzordnung zur Berechnung des charakteristischen Exponenten der Mathieuschen Differentialgleichung. Numer. Math. 27, 53-65 (1976)

23. Wagenfuihrer, E.: Die Determinantenmethode zur Berechnung des charakteristischen Exponenten der endlichen Hillschen Differentialgleichung. Numer. Math. 35, 405-420 (1980)

24. Wagenführer, E., Lang, H.: Berechnung des charakteristischen Exponenten der endlichen Hillschen Differentialgleichung durch Numerische Integration. Numer. Math. 32, 31-50 (1979)

25. Wagenführer, E.: Zur Eigenwertberechnung bei der endlichen Hillschen Differentialgleichung. Regensburg, 1980 (unpublished)

26. Weinstein, A., Stenger, W.: Intermediate Problems for Eigenvalues. New York, London: Academic Press, 1972

Received July 26, 1981/October 8, 1982 